Author(s):

\section{Submitted to:}

\section{MASTER

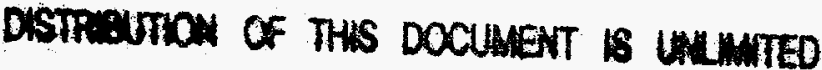

Report to funding Agency/Joint Program office for Biological Defense

\section{Los Alamos}

\section{NATIONAL LABORATORY}

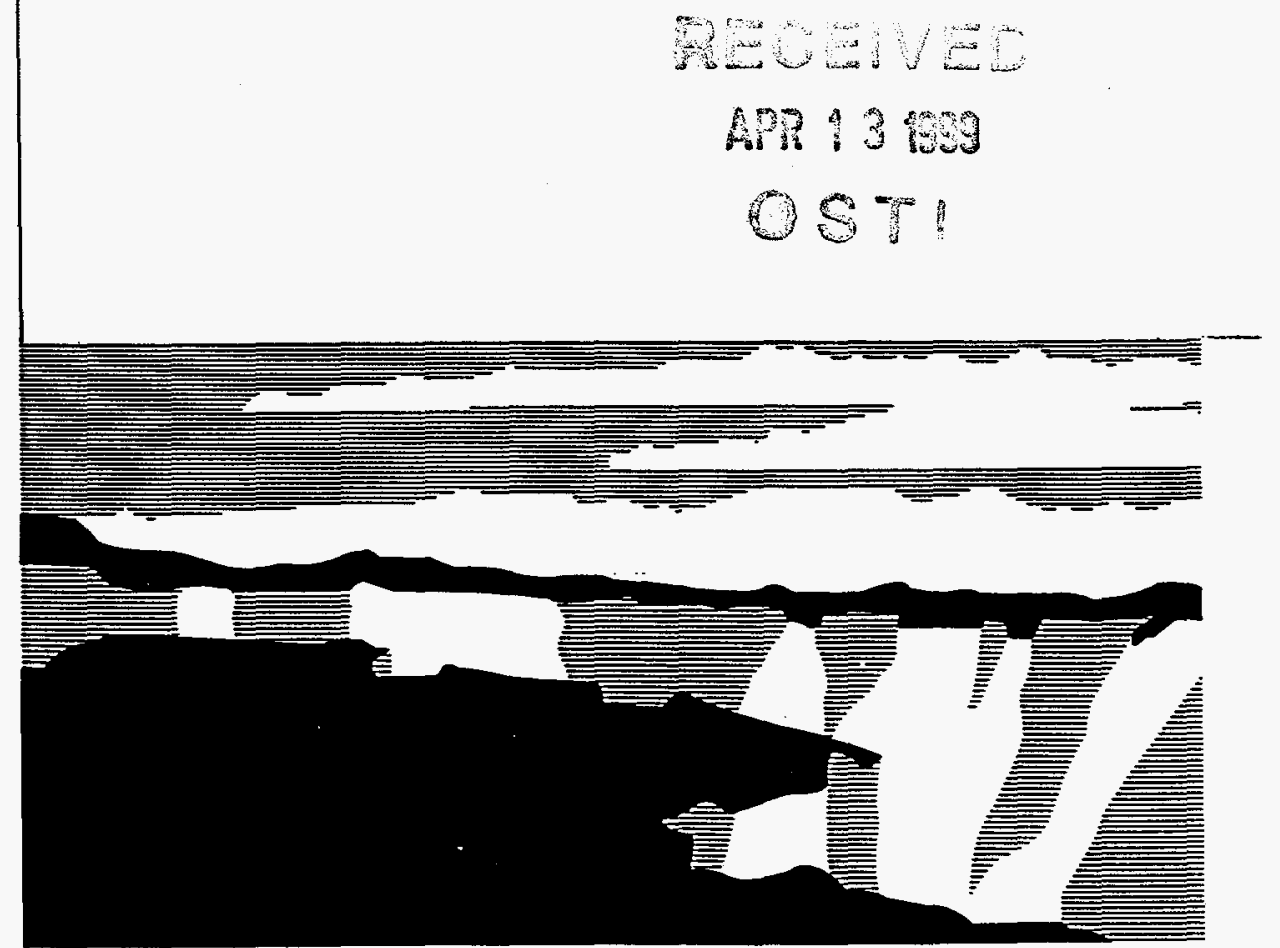

Los Alamos National Laboratory, an affimative actionequal opportunity employer, is operated by the University of Califomia for the U.S. Department of Energy under contract W-7405-ENG-36. By acceptance of this article, the publisher recognizes that the U.S. Govemment retains a nonexclusive, royalty-free license to publish or reproduce the published form of this contribution, or to allow others to do so, for U.S. Government pupposes. Los Alamos National Laboratory requests that the publisher Identify this article as work performed under the auspices of the U.S. Department of Energy. The Los Alamos National Laboratory strongly supports academic freedom and a researcher's right to publish; as an institution, however, the Laboratory does not endorse the viewpoint of a publication or guarantee its technical correctness. 


\section{DISCLAIMER}

This report was prepared as an account of work sponsored by an agency of the United States Government. Neither the United States Government nor any agency thereof, nor any of their employees, makes any warranty, express or implied, of assumes any legal liability or responsibility for the accuracy, completeness, or usefulness of any information, apparatus, product, or process disclosed, or represents that its use would not infringe privately owned rights. Reference berein to any specific commercial product, process, or service by trade name, tradernark, manufacturer, or otherwise does not necessarily constitute or imply its eadorsement, recommendation. or favoring by the United States Government or any ageacy thereof. The views and opinions of authors expressed herein do not necessarily state or reflect those of the United States Government or any agency thereof. 


\section{DISCLAIMER}

Portions of this document may be illegible in electronic image products. Images are produced from the best available original document. 


\title{
QUICK-LOOK EYE-SAFETY ASSESSMENT FOR THE SHORT RANGE LIDAR
}

\author{
Thomas R. Wehner \\ Los Alamos National Laboratory \\ July 2, 1998
}

\begin{abstract}
SUMMARY: This is a quick-look eye-safety assessment for the Short Range (SR) lidar, a system under development for standoff biological aerosol detection in the outdoor environment. The ground-vehicle-mounted SR lidar system will scan a sector of the nearby atmosphere with a repetitively pulsed, multiple-wavelength, UV/IR laser beam. This laser is not intrinsically eyesafe, and hence the SR lidar system requires a protection system to minimize the risk of eye exposures above the ANSI-standard maximum permissible exposure within a nominal hazard zone. The nominal ocular hazard distance for the UV/IR laser itself was calculated to be $6 \mathrm{~km}$. The protection system, which will include a scan-stop detector and a laser beam path interrogator, currently is conceptual only. Until the complete protection system is designed, evaluated, and tested, and a more detailed safety assessment has been performed, the eye-safety issue for the SR lidar system cannot be resolved.
\end{abstract}

INTRODUCTION: The SR lidar system is designed to be ground-vehicle mounted and to scan in azimuth and elevation many kilometers out into the atmosphere to locate aerosol clouds and UV-laser-induced fluorescence from atmospheric aerosols. The SR lidar system is planned to utilize two lasers, one a range finder, which is assumed to be commercially available off the shelf and accepted as proven eye-safe, and the other a special UV/IR laser built by Fibertek, Inc. Because personnel can be within the ocular hazard distance for the UV/IR laser, the SR lidar system concept incudes a protection system.

The proposed protection system has two principal elements. One is an engineered system that "interrogates" the UV/RR laser beam path with the range-finder laser. If this laser interrogation indicates that there is an object within the nominal hazard zone (NHZ) along the UV/IR laser beam path, then the UV/IR laser is not allowed to fire. If there is not an object in the path, then the UV/IR laser is allowed to fire. (Note: the system currently being tested at the Dugway Proving Ground does not include the range-finder laser. Strict adherence to approved SOPs in the controlled testing environment of DPG is the current principal means for providing eye protection and safety.)

Another principal element is a scan-stop detection system. When this system detects that scanning has stopped or is not functioning properly, the UV/IR laser is turned off. Other engineered safety features such as interlocks can be expected to also be part of the protection system. The complete protection system has not yet been designed.

The UV/IR laser operates in two separate modes. In one mode, the emergent beam is at $266 \mathrm{~nm}$, and in the other mode, the emergent beam has two wavelengths, $355 \mathrm{~nm}$ and $1570 \mathrm{~nm}$. (UV/IR-laser-specific data is given in Appendix A.)

NOMINAL HAZARD ZONES: Using the ANSI-Z136.1 standard, we calculated the nominal ocular hazard distance (NOHD) for the UV/IR laser and for the SR lidar system with specific assumptions. For the laser itself, we used the ANSI-standard 10- 
second viewing or stare time, and calculated the NOHD for each of the three wavelengths separately. Of the three wavelengths, we found the $266 \mathrm{~nm}$ wavelength to have the most restrictive (largest) NOHD. Without atmospheric attenuation, we calculated the NOHD for the $266 \mathrm{~nm}$ wavelength laser to be $6 \mathrm{~km}$ from the laser source, or in other words, the nominal hazard zone (NHZ) extends from the laser out to $6 \mathrm{~km}$. ${ }^{*}$ Thus, protective measures of some kind need to be employed in order to protect the eyes of personnel within $6 \mathrm{~km}$ from the laser. Beyond $6 \mathrm{~km}$ no protection is needed. Note that this 6-km result is for the UV/IR laser alone, not the SR lidar system which is to include a protection system. Also note that this would be the NOHD for the SR lidar system with failures of the beam path interrogator and the scan-stop detector.

The SR lidar system concept proposes a two-principal-element engineered protection system described above. If the SR lidar system is operating normally and scanning at reasonable rates (see Appendix A), and a person were to be exposed to a single UV/IR laser pulse, we calculated the nominal ocular hazard distance (NOHD) to be about 200 meters without atmospheric attenuation. Note that this would be the NOHD for the SR lidar system with a failure of the proposed beam path interrogator.

If the protection system for the SR lidar system works as proposed, then the risk of potentially eye-damaging exposures will be minimized. However, the question of whether the protection system as proposed will work properly cannot be answered until after design, evaluation, and testing. A more detailed safety assessment must be performed to quantify protection system failure rates and to determine whether the SR lidar system can be engineered to be "acceptably safe" for military use.

SYSTEM SAFETY ASSESSMENT NEEDS: Knowledge of the nominal hazard zone (NHZ) alone is not sufficient for a safety assessment for the SR lidar system. Additional information about the protection system and other engineered safety systems is required. Without this information a safety assessment will be incomplete. The information needed to complete the safety assessment will include two principal focus areas: the range-finder-laser beam-path interrogator system and the UV/IR laser scan-stop detection system. Other basic information, such as scan rate, is also needed.

We do not know of a laser-beam-path interrogator-type protection system that has already been utilized for other outdoor-use lasers. If such a system exists, the safety and operational experience from that system would support the safety assessment for the SR lidar system. Regardless, a determination of the safety and reliability of the SR lidar system cannot be made without at least a protection system design. When the design becomes available, we would suggest posing the following questions in the process of assessing the safety of the system.

First, how will the system assure that the spots (along the beam path) interrogated with the range-finder laser are the same spots the UV/IR laser would hit. The issue here revolves around the expectation that the range-finder laser beam and the UV/IR laser beam will not be co-axial. In a non-coaxial configuration, it is easier to encounter the situation in which the range-finder laser reports that no solid objects are "in the way," yet the UV/TR laser beam, because it is not traversing the exact same path as the rangefinder laser, does indeed strike a solid object that might happen to include a person's eyes. Possibly, the range-finder laser will have a larger divergence than the UV/IR

\footnotetext{
* The commercially available laser safety computer code, LAZAN, was also run for verification. The NOHD from LAZAN results was also $6 \mathrm{~km}$. Note that this distance is very conservative. If even a modicum of atmospheric attenuation is included, then the NOHD becomes $2 \mathrm{~km}$ (see Appendix A) which is consistent with CBDCOM results. However, though we can expect that most of the time a $2 \mathrm{~km}$ NOHD would be sufficient, operations could occur in situations in which the NOHD could be up to $6 \mathrm{~km}$, though never greater than $6 \mathrm{~km}$.
} 
laser and the range-finder laser spot will be large enough at distances of interest to encompass the smaller UV/IR laser spot even with slight aiming errors. If this is the case, it needs to be verified after the design is established.

Next, what is the mechanism for stopping the firing of the UV/IR laser when a solid object is detected with the range-finder laser? Ideally, for maximum safety, we would prefer this mechanism to be a direct link that uses the minimum number of physical processes only. If an additional circuit board is required, this would be expected to be less reliable than a direct method. (There are simply more "links in the chain" and some of them could be weaker than those in the shorter "direct chain.") If software is also required, then the reliability of the software comes into question as well, and would also require evaluation for safety and reliability. Furthermore, the mechanism must be relatively fast and work at greater than $30 \mathrm{hz}$, the pulse rate for the UV/IR laser.

One of the techniques ascribed to the SR lidar system for eye safety we have heard about is the technique of using the range-finder laser to map out the terrain (azimuth and elevation) at distance from the laser and requiring all firings of the UV/IR laser to be above that terrain. The safety questions that arise include the following. What is the mechanism for limiting the laser scan so that it does not go below the established limits from the mapping? If it is mechanical, how are the limits set, and what happens when the vehicle on which the laser is mounted shakes or is moved? If the mechanism is mechanical-electrical (such as a limit switch) how is the mechanical-electrical device set up and what keeps it in place? And again, the same questions about vehicle vibration and movement need to be addressed. If the mechanism includes software, what actuators and sensors transmit the elevation and azimuth to the software and what is their response time?

Scan-stop protection systems are fairly common, and so, the safety assessment for this system should be relatively quick and straightforward.

These are only some of the questions that need to be addressed in a SR lidar system safety assessment. For this assessment, more information on the modes of operation, interlock systems, the two principal protection systems, and other safety systems, is needed. The UV/IR laser in the SR lidar system is not intrinsically eye-safe, but that does not mean that the SR lidar system cannot be made "safe enough to be acceptable" and of benefit to US military commands.

RECOMMENDATIONS: We would suggest the following. After the protection system is designed, a preliminary safety/hazard assessment can be performed. If the assessment is satisfactory, then, after design evaluation and components testing, a full probabilistic safety/risk assessment or similar safety/hazard assessment should be performed and include a determination of the probability and risk of exposures above the ANSI-standard maximum permissible exposures (MPEs). The proper military review organization should then examine the assessment and determine whether the SR lidar system is acceptably safe. Last, the impact of the protection system on normal operations should be examined, and if it has not already been done a skin hazard assessment should be performed. 


\section{Appendix A: Determination of Nominal Ocular Hazard Distances}

We calculated the nominal ocular hazard distance (NOHD) separately for each of the three wavelengths using the UV/IR laser specification data from the table below. Because the 355 and $1570 \mathrm{~nm}$ wavelengths are emitted simultaneously, their effects are at least additive, and they could be synergistic. These effects were not explored in this quick-look assessment. All calculations use the "American National Standard for Safe Use of Lasers," ANSI Z136.1-1993, which we understand is accepted for both military and civilian safe practice. According to this standard, the UV/IR laser is a Class 4 laser, and a determination of the nominal hazard zone (NHZ) is required.

We give both the results for no atmospheric attenuation and limited attenuation representative of very clear sea-level type atmospheric conditions using the values shown in Table A-2. The more conservative results are the ones without atmospheric attenuation which give the maximum NHZ. The results with atmospheric attenuation are still conservative as the attenuation coefficients are for very clear conditions which probably do not occur very often. Of course, if the laser is being operated at higher altitudes or in a region of the world that has extremely clear atmosphere, then the calculated NHZs might be underestimates. For eye-safety considerations, the unattenuated values are preferred.

We have calculated both single and multiple pulse nominal ocular hazard distances (NOHDs). The multiple pulse calculations are for a 10 second exposure. The rationale for this exposure time from the ANSI-Z136.1 standard is that a 10 second exposure is to be used for invisible wavelengths because at these wavelengths there is no natural aversion response, that is, there is nothing visible that would cause a person to blink or turn away, but that eye movements provide a natural exposure limit that normally would not exceed 10 seconds. This duration of exposure would require that the SR lidar system experience either 1) simultaneous failures of the beam-path interrogator and the scan-stop detector, or 2) failure of the beam-path interrogator combined with personnel movement cross-range that exactly matched the scan rate for the UV/IR laser. Though these scenarios are not very likely, we cannot say that they are impossible.

If scanning continues and only the beam-path interrogator fails, then consideration of a single pulse is appropriate for calculation of the nominal ocular hazard distance (NOHD) as long as the scan rate is not too slow and the target person is not too close to the laser source. We do not know the scan rate for the SR lidar system. But we calculated that for the 0.2 mrad divergence beam if the scan rate is 1 degree per second or faster, then objects at 110 meters and farther away from the laser source would receive one pulse or a fraction of a pulse. (The CP lidar scan rate was originally designed to be variable between 0.5 and 3.3 degrees per second, and so, the 1 degree per second rate appears reasonable for estimation purposes.) At closer distances, an object could get more than one pulse at the 1 degree per second scan rate. As long as calculated NOHDs are beyond about 100 meters, then the single-pulse treatment is conservative, and that was the case herein. Regardless, the scan rate must be known for a more complete safety analysis. 
Table A-1. UV/IR laser specification data used in the assessment. ${ }^{*}$

\begin{tabular}{|r|r|r|r|}
\hline wavelength (nm) & 266 & 355 & 1570 \\
\hline pulse repetition rate (hz) & 30 & 30 & 30 \\
\hline pulse width (ns) & 22 & 26 & 29 \\
\hline energy per pulse (mJ) & 111 & 107 & 88 \\
\hline full-angle divergence (mrad) & 0.2 & 0.2 & 1 \\
\hline diameter of emergent beam (cm) & 6 & 6 & 6 \\
\hline scan angle limits & unknown & unknown & unknown \\
\hline scan rate & unknown & unknown & unknown \\
\hline
\end{tabular}

Table A-2. Atmospheric attenuation coefficients used in the assessment for typical, very clear air at sea level. ${ }^{\dagger}$

\begin{tabular}{|l|l|l|l|}
\hline wavelength $(\mathrm{nm})$ & 266 & 355 & 1570 \\
\hline coefficient $\left(\mathrm{km}^{-1}\right)$ & 1 & 0.1 & 0.04 \\
\hline
\end{tabular}

\section{$\underline{266 \mathrm{~nm} \text { Laser }}$}

Single Pulse:

According to Table 5 of the ANSI-Z136.1 standard, the maximum permissible exposure (MPE) for this wavelength is $3 \times 10^{-3} \mathrm{~J} / \mathrm{cm}^{2}$ for a single pulse of $22 \mathrm{~ns}$. With equation B12 from the ANSI-Z136.1 standard, we calculate the nominal ocular hazard distance (NOHD) to be 167 meters. So, without atmospheric attenuation, the nominal hazard zone (NHZ) is out to 167 meters from the laser source. To be most conservative, protective measures would be needed within this 167-meter distance, but not beyond, for this wavelength. This is the maximum NHZ possible as atmospheric attenuation has been neglected.

If we now include atmospheric attenuation, the nominal ocular hazard distance (NOHD) is reduced to 120 meters.

Multiple Pulses:

Exposures at this wavelength are additive for repetitive pulses. We assume a 10second stare or viewing time, during which a person looking toward the laser would be exposed to 300 pulses, for a total of $6.6 \times 10^{-6} \mathrm{~s}$ of beam-on exposure time and a total energy of $33.3 \mathrm{~J}$. The maximum permissible exposure (MPE) remains at $3 \times 10^{-3}$ $\mathrm{J} / \mathrm{cm}^{2}$ from Table 5 of the ANSI-Z136.1 standard. With equation B12 from the ANSI-Z136.1 standard, we calculate the nominal ocular hazard distance (NOHD) to be about $6 \mathrm{~km}$. So, the nominal hazard zone (NHZ) is out to $6 \mathrm{~km}$ from the laser source. To be most conservative, protective measures would be required within this $6-\mathrm{km}$

* Data sources: e-mail from LTC Bob Ranhofer, Joint Program Office for Biological Defense; phone conversations with Theresa Lewis of the Bio Standoff Group, CBDCOM, and with Mark Pronko and Ralph Burnham of Fibertek, Inc.

$\dagger$ Data from various sources including: 1) Handbook of Lasers, Robert J. Pressley, ed., Chemical Rubber Co., 1971, 2) Laser Beam Propagation in the Atmosphere, Hugo Weichel, SPIE Optical Engineering Press, 1990, 3) Laser Beams in the Atmosphere, V. E. Zuev, Consultants Bureau, 1982. 
distance, but not beyond, for this wavelength. This is the maximum NHZ possible as atmospheric attenuation has been neglected.

If we now include atmospheric attenuation, the nominal ocular hazard distance (NOHD) is reduced to $2.1 \mathrm{~km}$.

The exposure as a function of distance and the maximum permissible exposure (MPE) for the multiple-pulse assessment with and without atmospheric attenuation are shown in the figure below.

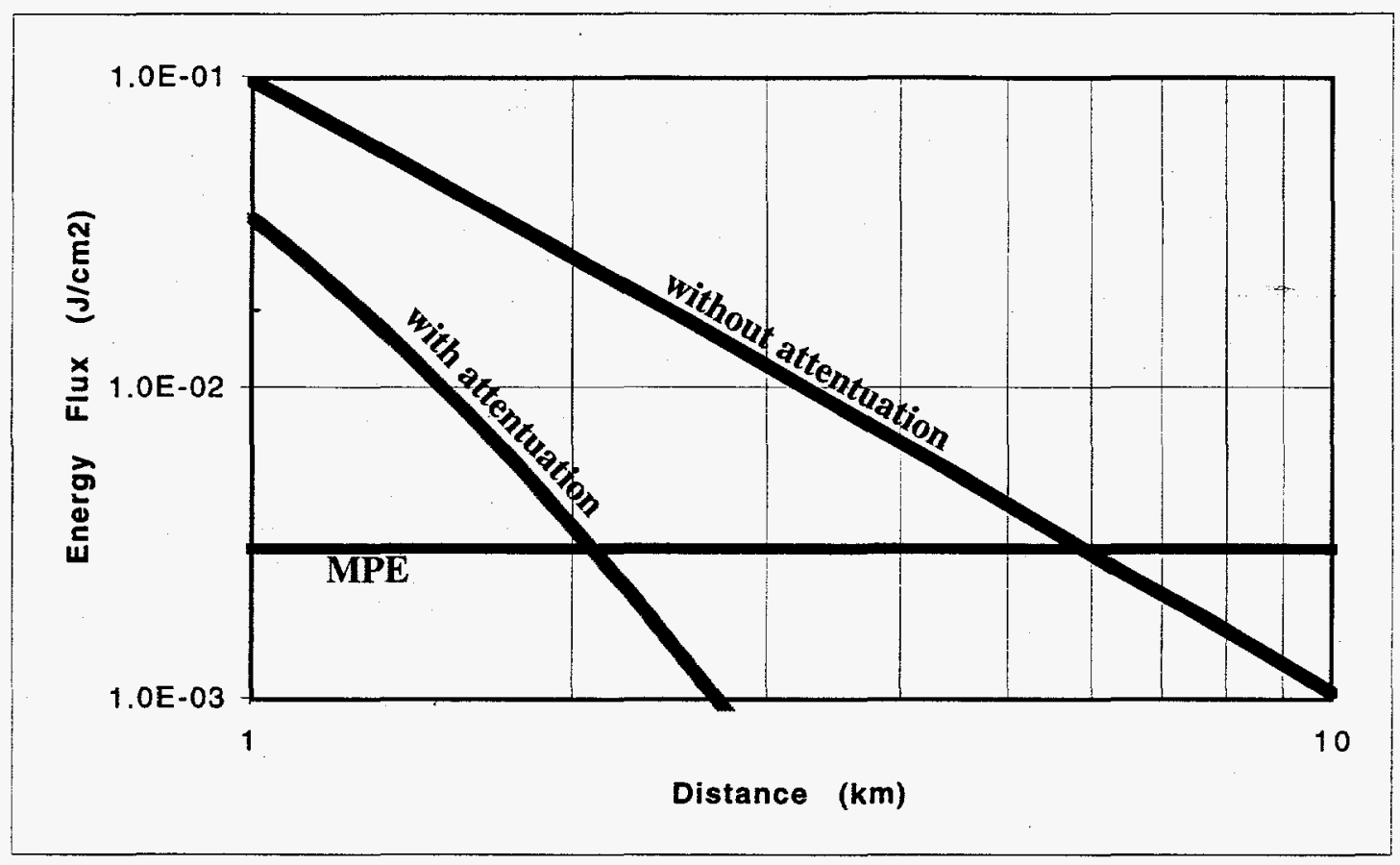

Figure A-1. The laser exposure or energy flux for a 10 -second stare vs. distance from the $266 \mathrm{~nm}$ wavelength laser source. The nominal ocular hazard distance (NOHD) without atmospheric attenuation is $6 \mathrm{~km}$, and with is about $2 \mathrm{~km}$, as shown by the intersections of the two curves with the maximum permissible exposure (MPE) line.

Summary:

If the stare or viewing time is 10 seconds or less, then to ensure that the eyes of personnel are not harmed in the unexpected case in which all the laser pulses during this time enter the eye, protective measures are needed within $6 \mathrm{~km}$ of the laser operating at this $266 \mathrm{~nm}$ wavelength. With atmospheric attenuation, this distance drops to $2.1 \mathrm{~km}$. If the stare time is longer the nominal hazard zone (NHZ) would increase.

For a single pulse, the NODH is 167 meters without atmospheric attenuation, and 120 meters with. 
Single Pulse:

According to Table 5 of the ANSI-Z136.1 standard, the maximum permissible exposure (MPE) for this wavelength is $7 \times 10^{-3} \mathrm{~J} / \mathrm{cm}^{2}$ for a single pulse of $26 \mathrm{~ns}$. With equation B12 from the ANSI-Z136.1 standard, we see that the nominal ocular hazard distance (NOHD) is zero, that is there is no nominal hazard zone (NHZ) at this wavelength for a single pulse.

If we include atmospheric attenuation, there is surely not a nominal hazard zone (NHZ) at this wavelength for a single pulse.

Multiple Pulses:

Exposures at this wavelength are additive for repetitive pulses. We assume a $10-$ second stare or viewing time, during which a person looking toward the laser would be exposed to 300 pulses, for a total of $7.8 \times 10^{-6} \mathrm{~s}$ of beam-on exposure time and a total energy of $32.1 \mathrm{~J}$. The maximum permissible exposure (MPE) is $3 \times 10^{-2} \mathrm{~J} / \mathrm{cm}^{2}$ from Table 5 of the ANSI-Z136.1 standard. With equation B12 from the ANSIZ136.1 standard, we calculate the nominal ocular hazard distance (NOHD) to be about $1.8 \mathrm{~km}$. So, the nominal hazard zone (NHZ) is out to $1.8 \mathrm{~km}$ from the laser source. To be most conservative, protective measures would be required within this $1.8-\mathrm{km}$ distance, but not beyond, for this wavelength. This is the maximum NHZ possible as atmospheric attenuation has been neglected.

If we now include atmospheric attenuation, the nominal ocular hazard distance (NOHD) is reduced to $1.7 \mathrm{~km}$.

Summary:

If the stare or viewing time is 10 seconds or less, then to ensure that the eyes of personnel are not harmed in the unexpected case in which all the laser pulses during this time enter the eye, protective measures are needed within $1.8 \mathrm{~km}$ of the laser operating at this $355 \mathrm{~nm}$ wavelength. If the stare time is longer the nominal hazard zone (NHZ) would increase.

For a single pulse, there is not a nominal hazard zone (NHZ) at this wavelength.

\section{$1570 \mathrm{~nm}$ Laser}

Single Pulse:

According to Table 5 of the ANSI-Z136.1 standard, the maximum permissible exposure (MPE) for this wavelength is $1 \mathrm{~J} / \mathrm{cm}^{2}$ for a single pulse of $29 \mathrm{~ns}$. With equation B12 from the ANSI-Z136.1 standard, we see that the nominal ocular hazard distance (NOHD) is zero, that is, there is no nominal hazard zone (NHZ) at this wavelength for a single pulse.

If we include atmospheric attenuation, there is surely not a nominal hazard zone (NHZ) at this wavelength for a single pulse. 
Multiple Pulses:

We assume a 10 -second stare or viewing time, during which a person looking toward the laser would be exposed to 300 pulses, for a total of $8.7 \times 10^{-6} \mathrm{~s}$ of beam-on exposure time and a total energy of $26.4 \mathrm{~J}$. At this wavelength the single pulse maximum permissible exposure (MPE) is the same as the CW MPE for a 10-second exposure times the smaller of the $\mathrm{C}_{\mathrm{p}}$ correction factor or one over the number of pulses. Thus', the MPE is $3.3 \times 10^{-3} \mathrm{~J} / \mathrm{cm}^{2}$ from Table 5 of the ANSI-Z136.1 standard. With equation B12 from the ANSI-Z136.1 standard, we see that the nominal ocular hazard distance (NOHD) is zero, that is, there is no nominal hazard zone (NHZ) at this wavelength for a single pulse.

If we include atmospheric attenuation, there is surely not a nominal hazard zone (NHZ) at this wavelength for a multiple pulses.

Summary:

If the stare or viewing time is 10 seconds or less, then protective measures are not needed at this $1570 \mathrm{~nm}$ frequency. At some stare time longer than 10 seconds, a nominal hazard zone (NHZ) would develop, and its extent would depend on the stare time.

For a single pulse, there is not a nominal hazard zone (NHZ) at this wavelength.

\section{Overall Summary}

The nominal ocular hazard distances (NOHDs) for each of the laser wavelengths has been calculated separately, and are shown in the table below. Additive and synergistic effects, if any, between the simultaneous $355 \mathrm{~nm}$ and $1570 \mathrm{~nm}$ wavelengths have not been examined. For the overall system, protective measures would be required within $6 \mathrm{~km}$ from the laser system location. If anticipated or expected accidental viewing or stare times were to increase beyond 10 seconds, then the NOHD would also increase.

Table A-3. Calculated nominal ocular hazard distances (NOHDs).

\begin{tabular}{|c|c|c|c|}
\hline wavelength (nm) & 266 & 355 & 1570 \\
\hline single-pulse NOHD (m) & 120 to 170 & 0 & 0 \\
\hline multiple-pulse NOHD $(\mathrm{km})$ & 2 to 6 & 1.7. to 1.8 & $\overline{0}$ \\
\hline
\end{tabular}




\section{Appendix B: Other Recommendations}

Only the nominal hazard zone (NHZ) for intra-beam viewing was calculated for the UV/IR laser in this quick-look assessment. In a more comprehensive assessment, other calculations should be performed. These include considerations of transmission through windows, specular reflections, diffuse reflections, and optically aided viewing including with.binoculars and night vision goggles.

Because the SR lidar system could penetrate navigable airspace with its laser beams, operation of the SR lidar system domestically would require coordination with the FAA. Neither this nor the military equivalent was investigated herein. In a more comprehensive safety assessment this aspect should be addressed.

The ANSI-Z136.1 standard is fairly explicit with respect to invisible laser emissions, which all three SR lidar system UV/IR laser wavelengths are, and states in section 4.5.1.2, "The general public shall not be exposed nor have access to laser radiation emission at wavelengths outside the visible range $(0.4$ to 0.7 microns $)$ at levels exceeding the applicable MPE levels under any possible conditions of operation." The applicability of this statement to the proposed protection system needs to be explored in a more detailed safety assessment.

The military medical community should be consulted. This community has experience with laser eye injuries and can probably offer valuable information and recommendations in the more detailed safety assessment. In the 1997 paper at the International Laser Safety Conference entitled "Accidental Human Retinal Injuries by Laser Exposures: Implications to Laser Safety," B. E. Stuck et al. of the Walter Reed Army Institute of Research note that the military owns large numbers of laser rangefinders, designators and training devices and uses them in outdoor environments, but that the number of accidents or incidents has been relatively few. They state that, "Proactive laser safety training and range control procedures have been effective in minimizing the number of eye injuries." However, they also caution that, "Uncontrolled military operations will probably result in increased number of injuries" and they suggest that "Better eye protection...regimens are required to minimize vision loss from such exposures."

Last, operator and maintenance personnel eye protection and safety should be addressed also. The design should be robust against stray light to offer the maximum protection for operators who may not be very knowledgeable about lasers. Additional safety measures should be included to protect those doing maintenance on the system as well. So, the safety assessment should include maintenance and operations functions also. 